\title{
The study of spectrum reconstruction based on fuzzy set full constraint and multiendmember decomposition
}

\author{
Yanbiao Sun ${ }^{\mathrm{a}}$; Yi Lin ${ }^{\mathrm{b}}$; Xingbang Hü ${ }^{\mathrm{b}}$; Shuaiyang Zhao ${ }^{\mathrm{b}}$; Suihua Liu ${ }^{\mathrm{c}}$; Qingxi Tongd ${ }^{\mathrm{d}}$ Helder Dennis ${ }^{\mathrm{e}}$; Lei Yan $^{\mathrm{b} *}$ \\ ${ }^{a}$ Chinese Academy of Surveying \& Mapping, Beijing 100039, PR China - syb51@163.com \\ bInstitute of Remote Sensing and GIS, Peking University, Beijing 100871, PR China - (yi.lin, huxingbang, syzhao, \\ lyan)@pku.edu.cn \\ c Guizhou Normal University, Guiyang, Guizhou 550001, PR China - 1sh23h@163.com \\ d Institute of Remote Sensing and Digital Earth, Chinese Academy of Sciences, PR China - tqxi@263.net \\ e South Dakota State University, South Dakota 57007, USA - Dennis.Helder@SDSTATE.EDU
}

Commission VI, WG VI/4

KEY WORDS: Multi-spectral; Hyper-spectral; Reconstruction; Fuzzy sets; Multi-endmember; Spectral library

\begin{abstract}
:
Hyperspectral imaging system can obtain spectral and spatial information simultaneously with bandwidth to the level of $10 \mathrm{~nm}$ or even less. Therefore, hyperspectral remote sensing has the ability to detect some kinds of objects which can not be detected in wide-band remote sensing, making it becoming one of the hottest spots in remote sensing. In this study, under conditions with a fuzzy set of full constraints, Normalized Multi-Endmember Decomposition Method (NMEDM) for vegetation, water, and soil was proposed to reconstruct hyperspectral data using a large number of high-quality multispectral data and auxiliary spectral library data. This study considered spatial and temporal variation and decreased the calculation time required to reconstruct the hyper-spectral data. The results of spectral reconstruction based on NMEDM showed that the reconstructed data has good qualities and certain applications, which makes it possible to carry out spectral features identification. This method also extends the application of depth and breadth of remote sensing data, helping to explore the law between multispectral and hyperspectral data.
\end{abstract}

\section{INTRODUCTION}

Hyperspectral remote sensing is widely used for ground exploration, hydrology, meteorology, oceanography, geology, and other aspects of forest research (Hunt, 1979). Unfortunately, there are relatively few hyper-spectral sensors among the civil satellites in orbit, and it is difficult and expensive to acquire hyper-spectral data for a region of interest (Tong Q-X, et al., 2006). There is a conflict between spatial and spectral resolution due to energy conservation, so hyper-spectral imaging tends to have lower spatial resolution over the actual course of a study. It is very difficult to calibrate tens of thousands of sensing elements, resulting in images that have a large number of bad lines, and most bands exist to varying degrees in many stripes. In addition, hyperspectral sensors experience significant performance degradation within a short time. These problems severely restrict use of hyper-spectral data (Chen Y-Y, 2008). In recent decades, high-quality multispectral data has become available, but has not been fully used. Multi-spectral sensors have a relatively stable spectrum performance through continuous scaling and can provide long-term reliable multispectral data.

In this paper, we developed a reconstruction method based on hyperspectral imaging and spectroscopy between multi-spectral and hyper-spectral images that can effectively overcome the shortcomings discussed above. Hyperspectral reconstruction based on a multispectral sensor can: (1) analyze the physical nature of the process of hyperspectral imaging and solve a mixed reconstruction of the dual relationship (Zhang L-F, 2005); (2) provide mixed pixel un-mixing reverse means; (3) use strong stability of a multi-parameter spectrometer to determine why hyper-spectral sensor parameters are easily degraded in the process; (4) determines the association between hyper-spectral and multi-spectral data; and (5) obtains high-quality hyperspectral data in the absence of real data and with incomplete knowledge of the ground. In addition, reconstructed hyperspectral image data can be a useful supplement to existing data, and synthetic hyperspectral, making it conducive to feature analysis and detection (Valero et al., 2007; Heikkinen et al., 2007)

The focus of this paper was the reconstruction of hyperspectral image data under the conditions of a fuzzy set (FS) of full constraints and normalized multiendmember spectral decomposition. We used the inverse process of unmixing to understand the nature of hyper-spectral imaging, based on the theory of the Normalized Multiple Endmember Decomposition

${ }^{*}$ Corresponding author 
(NMED) and FS operations. Starting from the multispectral image and spectral characteristics, through normalized multi-endmember decomposition the conditions of FSs were fully constrained, using the dual relationship of unmixing - reconstruction, to reconstruct the continuous spectrum curve. This expanded the spectral range and depth of mining for spectral information. Spectral reconstruction was undertaken with several classes of endmember using a representative and universal spectral library. On the basis of accurate unmixing, we considered the subtle temporal and spatial variations of endmembers, while at the same time improving the efficiency and reconstructing hyper-spectral data. This made it possible to obtain a continuous spectral reflectance from the multispectral image.

\section{METHOD AND MATERIALS}

\subsection{Method}

In the spectrum reconstruction model, it was assumed that each incident photon affected only a single pixel component ( Chen Y-Y, 2008; Imai et al., 2000). A signal $(\mathrm{R})$ consisting of mixed pixels can be a pure spectral component signal and the spectral weights (weights determined by the sub-pixel coverage) (Zhang L-F, 2005; Liu S-H, 2013; Luo B, 2010; Foppa et al., 2007) are described as follows. A multispectral image feature extraction spectrum can be expressed as the spectrum shape. The spectrum can be expressed as a linear combination of several kinds of standard spectral patterns and residual items:

$$
R=f_{1} e_{1}+f_{2} e_{2}+\cdots+f_{n} e_{n}+r
$$

where $R$ is the reflectivity of a pixel

$$
e_{1}, e_{2}, \ldots, e_{n} \text { are different components of }
$$

an actual scene, index is the number of endmembers

$$
f \text { is coverage, }
$$$$
r \text { is residual error }
$$

The multi-spectral sensor can be presented as:

$$
R_{M}=e_{M} f+r_{M}
$$

where $\mathrm{M}$ is the multi-spectral sensor. Endmember composition and their abundance ratio did not change in the hybrid scenario, and we obtained the coverage $f$ through the FS operations.

As long as we replaced $e_{M}$ with $e_{H}$, which was previously found by multiport decomposition in spectral libraries, we could obtain hyperspectral data that required reconstruction:

$$
R_{H}=e_{H} f+r_{H}
$$

where $H$ represents the hyper-spectral sensor $R_{H}$ is reconstructed hyper-spectral data

\subsection{Materials}

We reconstructed hyperspectral data from multispectral data. In terms of the quality and accessibility of hyperspectral data the choice is quite limited, but in this study we used Hyperion data. In contrast, there is a lot of data that can be used for multispectral data. To make the reconstruction as accurate as possible, we needed to consider multispectral and hyperspectral imaging data together as closely as possible. For this reason, we used Advanced Land Imager (ALI) multispectral data, which is the same platform as the Earth Observing One (EO-1) mission.

\section{RESULTS AND DISCUSSION}

We separately extracted the soil and vegetation endmember spectrum from the spectral library of water, and these endmember spectra were averaged as a mixed spectrum. We then reconstructed the soil and vegetation endmember spectrum by the UPDM and FSME models, respectively, in addition to the reconstruction to remove the uncalibrated and vapor absorption bands. The simulated spectrum and original spectrum were subjected to a comparative analysis. Figure 1 shows that the results for (a) water, (b) soil and two pure endmembers by the two reconstruction methods were relatively close. For (c) vegetation, and (d) two mixed spectral features, the FSME simulation results were significantly better than the UPDM results.

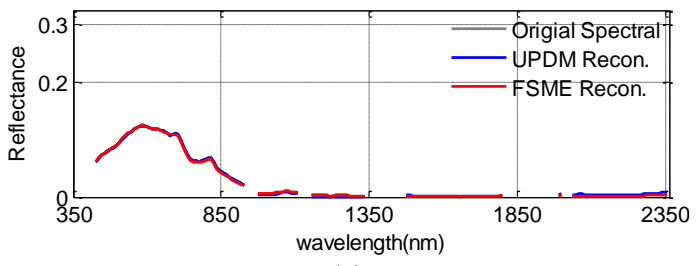

(a)

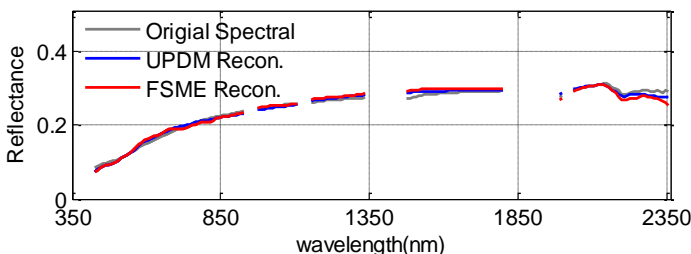

(b)

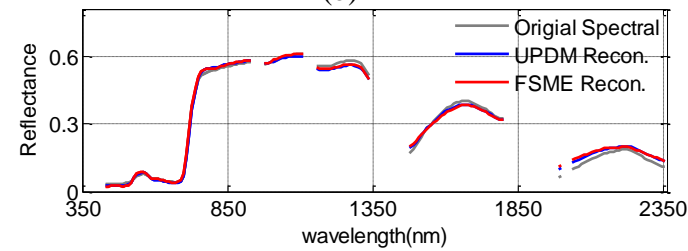

(c)

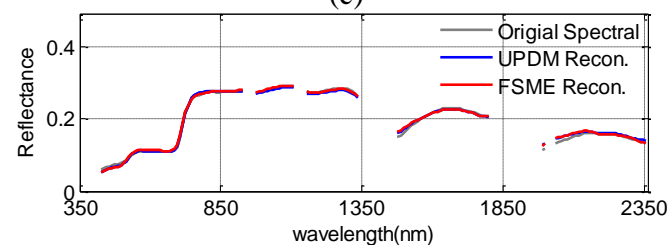

(d)

Figure 1. Original spectra (gray) and The Unified Profile for DoDAF and MODAF (UPDM) reconstruction (blue) and Fuzzy Set Multi-Endmember (FSME) reconstruction (red): (a) water (b) soil (c) vegetation (d) mixture 
Figure 2 shows a comparison of the corresponding residuals. Because water and soil has a low reflectivity, there is a very small difference between the two reconstruction methods. For the vegetation with higher reflectance spectra and the hybrid spectrum, the residual error plots clearly indicated that FSME produced better simulation results. In particular, it should be noticed in Figure 4 (c) that for the UPDM near $720 \mathrm{~nm}$, the position of the red edge had large residuals, but for FSME there were better reconstruction results, which did not produce this phenomenon.

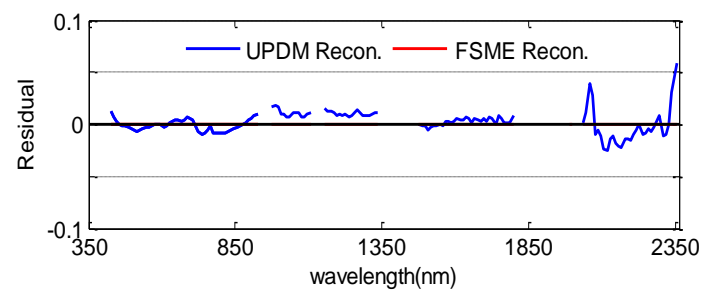

(a)

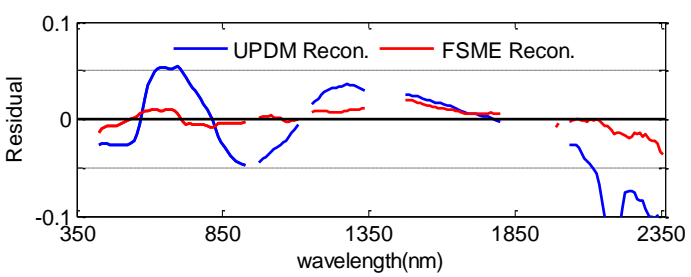

(b)

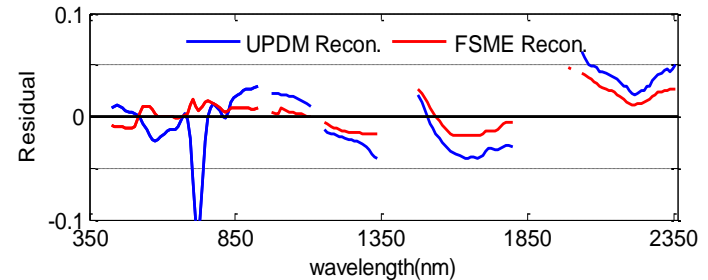

(c)

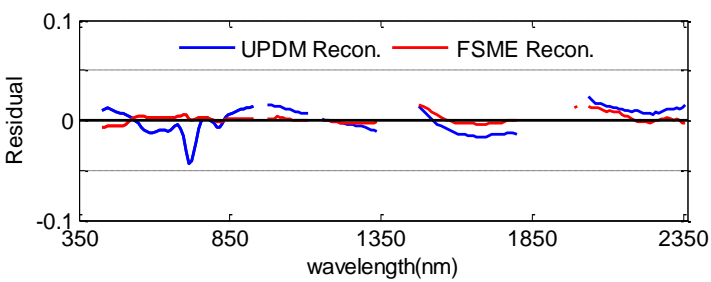

(d)

Figure 2. Comparison of the residual errors of UPDM (blue) and FSME (red): (a) water (b) soil (c) vegetation (d) mixture

Considering the root mean square error (RMSE) of the two reconstructed models for water, vegetation, soil, and mixed spectra (Table 1), it can be seen that FSME was significantly better than the UPDM. For vegetation, the RMSE of FSME was only 0.0161, less than the RMSE of the UPDM of 0.0317. For water, the RMSE of the FSME was approximately 0 , also better than the RMSE of the UPDM of 0.0021. For soil, the RMSE of the FSME was greater than the RMSE of the UPDM, reaching 0.0102 .

\begin{tabular}{lllll}
\hline & WATER & SOIL & VEGE & MIXTURE \\
\hline UPDM & 0.0021 & 0.0068 & 0.0317 & 0.0123 \\
FSME & 0 & 0.0102 & 0.0161 & 0.0048 \\
\hline
\end{tabular}

Table 1. Root mean square error (RMES) of the universal pattern decomposition method (UPDM) and Fuzzy Set Multi-Endmember (FSME) models

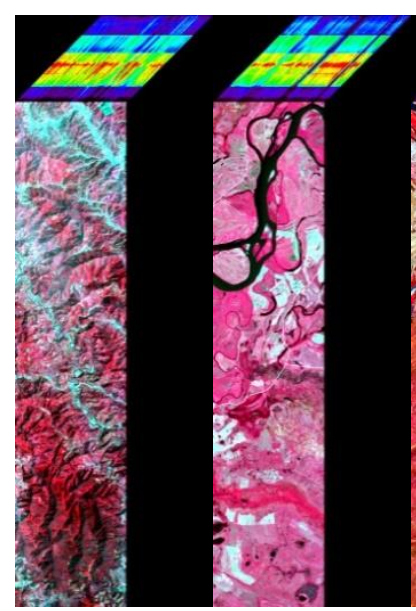

(a) (b)

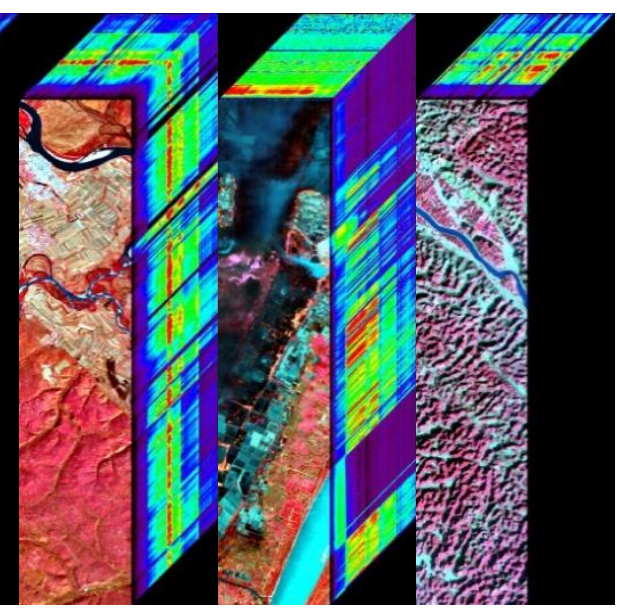

(d) (e) 


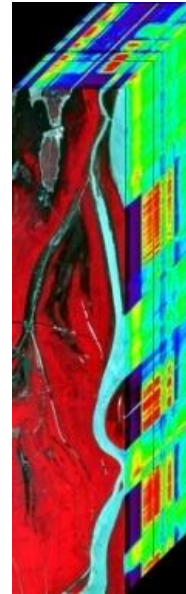

(f)

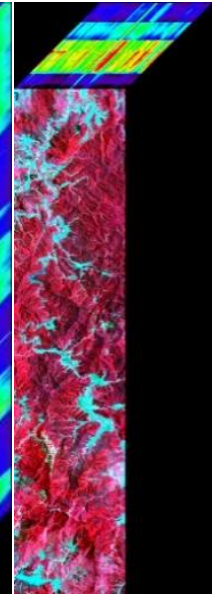

(g)

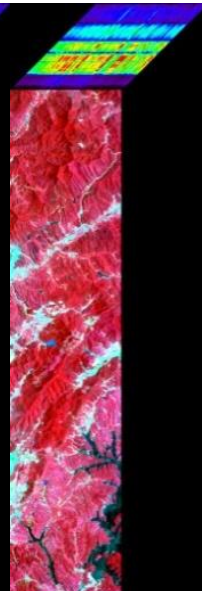

(h)

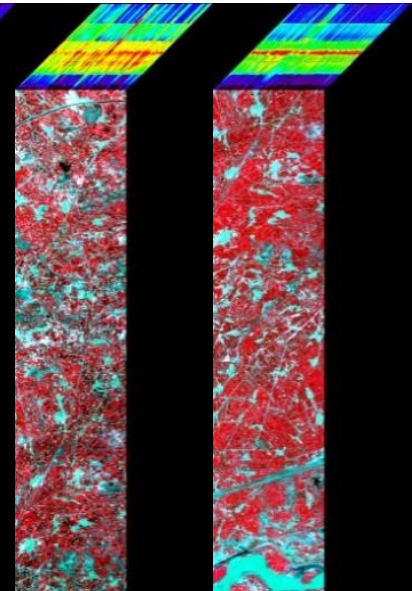

(i)

(j)

Figure 3 False color composite image of a reconstructed hyper-spectral cube: composite bands 43 (854 nm), 24 $(659 \mathrm{~nm})$, and $15(569 \mathrm{~nm})$.

(a) (j) are the following reconstructed products: EO1H1260432004281110PY, EO1H1260432003326110PZ, EO1H1180242004154110KY，EO1H1190242001271111PP，EO1H1230392011316110KF, EO1H1230402006226110KF, EO1H1210412009157110PX, EO1H1200402003211110KY, EO1H1290312003244110KP, and EO1H1290312003228110KP

For the reconstructed and original images, we choosed $43(854 \mathrm{~nm}), 24(659 \mathrm{~nm})$, and $15(569 \mathrm{~nm})$ composite false colors and $24(659 \mathrm{~nm}), 15(569 \mathrm{~nm})$ and 7 (487 $\mathrm{nm})$ composite true colors. Figure 3 presents false color composite images of a reconstructed hyper-spectral cube. For synthetic images, we can see the analog images and the original images have no significant difference; the two images of color, texture, shape, and boundaries were basically the same, which clearly shows that the characteristics and information of the original image remain in the analog image. Visually, the analog image is brighter and more brilliant than the original image, mainly because the analog video taken directly from the measured spectrum simulation was less affected by atmospheric and instrument noise. For a further analysis of the details, we also compared the similarities of the original and the reconstructed images for a single band. We randomly selected 7 (487.8679 $\mathrm{nm}), 43(854.1786 \mathrm{~nm}), 52(972.9932 \mathrm{~nm}), 68$ $(1134.3796 \mathrm{~nm}), \quad 81(1265.5618 \mathrm{~nm})$, and 147 $(2274.4175 \mathrm{~nm})$ bands, and found that 6 bands were quite similar, with the gray texture information being better. For the 52 and 68 bands, the bad lines and bands of the reconstructed image were eliminated. Overall, the reconstructed image contrast ratio was also stronger. The main reason may be that the reconstructed image was less affected by the atmosphere.

\section{CONCLUSIONS}

We propose a new reconstruction model-FSME, in which multispectral data is used to simulate hyperspectral data. This new model can be applied at the surface or using a portable spectrometer based on field or laboratory measurements of spectra obtained from a library or directly from the base of the spectrum image data. Only three end-members participated in the simulation and contributed to the spatial and temporal variability of endmembers in the simulation process. The results showed that our proposed reconstructed model was fully rational and the reconstructed image was considered to be good. Our conclusions are summarized as follows:

(1) Reconstruction of spectrum can be achieved according to the principles of science. The most important material basis is the construction of a spectral library. The ideal condition is to cover the construction of different times and a variety of typical spectral features in a spectral library on Earth. However, due to differences in surface features and imaging conditions, this is basically impossible to achieve. There is also an issue regarding the actual interference of the "synonyms spectrum" of remote sensing with the "spectrum foreign body" phenomenon.

(2) Another problem concerns scaling effects. The remote sensing image resolution scale is inconsistent with the observed spectral features, which was not considered in the study. This resulted in the study being disjointed, which also affected the efficiency of the spectral library.

(3) The spectral reconstruction model determines the physical principles of spectrum reconstruction and realization. The material basis of the spectral library reconstruction model is then the theoreticcally reconstructed physical spectrum. The spectral reconstruction model used in this study was a simple spectral model (SMA) based on the inverse model. It mainly used the reverse process solution mix for each pixel and, based on thousands of spectra, an iterative spectral library cycle determined endmember components and the end Genpo of the optimal linear fit to obtain the best reconstruction model. This model was simple and easy to implement and, with respect to the use of a standard spectrum (usually the average), the presence of the same class endmembers in different environments significantly improved the accuracy of the reconstruction. Based on the above, if the reconstruction error was too large, it 
may be due to the multiple scattering of a nonlinear reconstruction. However, it is clear that still not sufficient. The reconstruction range of the spectral reconstruction can span the entire optical remote sensing range $(0.3 \sim 2.5)$ for different applications, such as a reconstruction of the vegetation of the spectrum, the various features of its green reflection peak, and the red absorption edge position. Valley performance is clearly not the same and the sensitivity of each band is also not the same. In our reconstruction model, all of the bands were reconstructed in the same way, and there was no difference between the features under consideration.

(4) The reconstruction calculation is enormous. For example, in a three endmember reconstruction, if each class has a 100 endmember spectrum, the entire computing process needs to be $100 \times 100 \times$ 100 (i.e., 1,000,000) cycles to determine the optimal reconstruction model. If an image is 1,000 $\times 1,000$ pixels, it also needs $1,000,000$ cycles of calculation, which is clearly undesirable. Therefore, the model must be optimized and candidate reconstruction models must be screened for. As mentioned in the previous section, we can select the feature band or sensitive bands to reduce the number of bands required for reconstruction, thereby reducing the amount of data used in the calculation. In addition, it is possible to optimize the reconstruction of the model under different conditions to reduce the number of loop iterations. For such a large amount of computation, parallel computing should be used to improve computational efficiency.

This study used a large amount of high-quality multispectral data to reconstruct corresponding hyperspectral data. This reconstruction makes it possible to indirectly use the spectral waveform information from multispectral Earth observation data, with precise feature identification. This will expand the depth and breadth of applications of remote sensing data, and enable conversion of multi-spectral and hyper-spectral information. Spectral reconstruction is the reverse of the mixed pixel unmixing process, and is a novel procedure. In addition, the reconstruction of hyper- spectral remote sensing data for various applications has many positive uses, such as designing systems, understanding the image forming process, and the development and validation of data processing algorithms.

\section{ACKNOWLEDGEMENTS}

This study was supported in part by the National Natural Science Foundation of China, No. 41371492

\section{REFERENCES}

Chen Y-Y, 2008. Color spectrum reconstruction of object surface based on digital camera, $\mathrm{PhD}$ diss., Zhejiang Uni., Zhejiang Province., China.

Foppa, N., Hauser, A., Oesch, D., Wunderle, S., Meister, R., 2007. Validation of operational AVHRR subpixel snow retrievals over the European Alps based on ASTER data. International Journal of Remote Sensing 28, 4841-4865. doi:10.1080/01431160701253287

Hunt, G.R., 1979. Near-infrared (1.3 - 2.4 micrometre) spectra of alteration minerals - Potential for use in remote sensing. Geophysics 44, 1974-1986.

Imai, F., Berns, R., Tzeng, D., 2000. A comparative analysis of spectral reflectance estimated in various spaces using a trichromatic camera system. Journal of Imaging Science and Technology 44, 280---.

Tong Q-X, et al. 2006. Hyper-spectral remote sensingprinciples, technologies \& applications. Higher Education Press, Beijing, pp. 59-65.

Valero, E.M., Nieves, J.L., Nascimento, S.M.C., Amano, K., Foster, D.H., 2007. Recovering spectral data from natural scenes with an RGB digital camera and colored filters. Color Research and Application 32, $352-360$.

Zhang L-F, 2005. The Uneiversal Pattern Decomposition Method and the Vegetation Index Based on the UPDM, PhD diss., Wuhan Uni., Hubei Province., China. 